\title{
CASE ROLE FILLING AS A SIDE EFFECT OF VISUAL SEARCH
}

\author{
Heinz Marburger \\ Research Unit for Information Science \\ and Artificial Intelligence \\ University of Hamburg \\ Mittelweg 179 \\ D-2000 Hamburg 13, F.R. Germany
}

\author{
Wolfgang Wahlster \\ FB10 - Angewandte Mathematik \\ und Informatik \\ University of Saarbrücken \\ Im Stadtwald \\ U-6600 Saarbrücken 11, F.R. Germany
}

\begin{abstract}
This paper addresses the problem of generating communicatively adequate extended responses in the absence of specific knowledge concerning the absence of specific knowledge concerning the intensions of the questioner. We formulate and justify a heuristic for the selection of optional deep case slots not contained in the question as tained in an extended response. It is shown that. in a visually present domain of discourse. case role filling for the construction of an extended response can be regarded as a side effect of the visual search necessary to answer a question containing a locomotion verb. The paper describes the various representation constructions used in the German language dialog system HAM-ANS for dealing with the semantics of locomotion verbs and illustrates their use in generating extended responses. in particular. we outline the structure of the in particular, we outline the structure of the geometrical scene description, the representation tion language. the case-frame lexicon and the representation of the referential semantics based on the Flavor system. The emphasis is on a object-oriented programming methods for coping with the semantics of locomotion verbs. The process of generating an extended response is illus. trated by an extensively annotated trace.
\end{abstract}

\section{INTRODUCTION}

Frequently a questioner expects more than a direct. Iiteral response although he must assume that the answerer is not informed about what particular information he is seeking. The questioner imputes to a cooperative dialogue partner the communicative competence to reply to a simple yes-no question like (1) with an extended response (cf.

(1) Are you going to travel this summer?

(1a) Yes, to sicily.

In the absence of special information about the previous course of the dialog or the intentions of the questioner (the unmarked case) an answer like (1a) seems more appropriate than (1b) or (1c).

(ib) Yes, with an old school frierid.

(1c) Yes, by plane.

of course, there are numerous dialog situations in which (ib) or (ic) could be generated as a communicatively adequate response on the basis of a par. ticular partrier model. But it still must be asked why in dialogs of the type information upply the unmarked response takes the form (ta) ind not (1b) or $(1 \mathrm{c})$

In this paper we will present the results of a computational study of this problem for the domain

Research On HAM ANS is currently being supported by the German Ministry of Research and Technology (BMFT) under contract $081 T 15038$ 'locomotion verbs' in dialogs based on a visually present world of discourse. This question is parcooperative dialog systems, since, in many applications, no explicit knowledge about the dialog goals of the questioner is available at the outset. If a system is nevertheless expected to haver-answer if i,e. to volunteer information that has not specificaliy been requested, selecting the mand a set of heuristic criteria for selecting the adiitio.

It is noteworthy that the three additional points of information in (1a). (1b). (1c) correspond to filled deep case slots of the verb used in the question (GOAL CO-AGENT and INSTRUMENT, Tespecquestion this sugests that the unfilled optional case siots in the question are candidates for case slots in the question are candidates for in which all the deep case slots of 'break' are filled, only a direct response like $(2 a)$ is to be expected as a positive answer. While in ( 3 ), where extended response like (3a) can be expected.

(2) Did you break the window with your slingshot yesterday?

(2a) Yes.

(3) Did you break the window?

(3a) Yes, with my slingshot.

Since not every optional deep case of a given verb unspecified in the question is suitable for an unmarked extended response le.g. (1a)-(1c)) we may define the problem more precisely by asking which are to be chosen as the unmarked values.

For our domain of investigation 'locomotion verbs' let us consider questions (4) and (5). which refer to a visually present world of discourse. In each case perceptual processes are assumed as a prerequisite for the answer.

(4) Which vehicle stopped?

(4a) The bus, on Hartungstreet.

(4b) The bus, because the driver stepped on the brake.

(5) Did the bus turn off?

(5a) Yes, from Hartungstreet onto schlueterstreet. (5b) Yes, together with the taxi cab.

The instantiation of the locative slot in answer (4a) and the source and goal slots in (5a) is predictable in contrast to the causative slot, in (4) and (5) demonstrate, the same optional deep case slot is not always selected as the unmarked case slot is not always selected as the verb conoption. The choice is dependent upon the verb concombinations of deep cases are possible in combinations of deep cases
unmarked extended responses.

In the area under investigation here, the following heuristic car be employed to determine the 


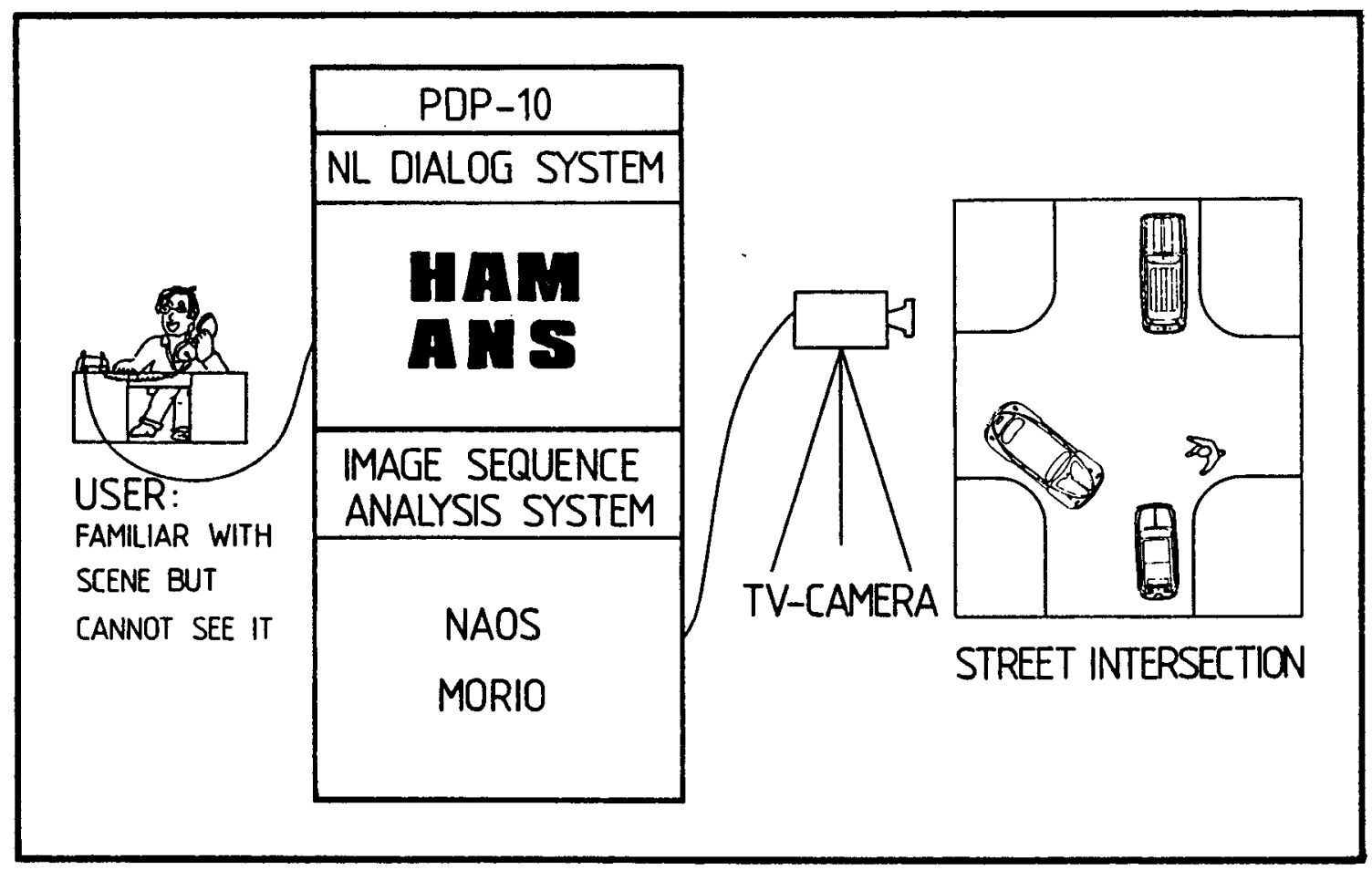

Fig. 1: Situational context of the dialog

selection of the deep case slots for an urimarked extended response: select the deep casc slots which contain the concepts necessary for the perceptual verification of the motion described by the verb.

In order to verify a stop-event it is necessary to determine the end point of the motion ( $c f .(4 a)$ ) but not the cause (cf. (4b)). For a turn-off event a change of direction between source and goal must a change of direction between source and goal must determine whether other objects make this change of direction at the same time (cf. (5b)).

Hence case rule filling for the construction of an extended respunse can be regarded as a side effect of the visual search necessary to answer the ques tion.

This also appears plausible when seen in the light of the beliefs that the questioner imputes to the answerer. The questioner believes that the answerer. The questioner believes that the answering the question and that it is therefore unnecessary to explicitly mention these in the unnecessary to explicitiy mention these in the question. Additionally the questioner believes expects an extended reply and fur this reason did not explicitily request the additional information. A cooperative dialog system fulfills this user expectation by applying the heuristic formulated above.

A prerequisite for the application of this heuristic is that the system liave knowledge about which deep case slots are relevant for the verification of a movemint. This prerequisite is not met by must natural language (NL) systems since they simply represent events in the domain of discourse in rully instantiated form using case frames, e.g. as nart of a sellintic net or frame hierarchy. In contrast, the Crrman language dialog system HAM-ANS (llamburg arplication.oriented natural language system] [6], which we have developed, can apply this heurishc because in addition to the case frame of each verb the system includes a represen. tation of the referential semantics of predica. tions associ ited with that verb which makes it possibie to t:valuate the visual input data for the movement in question.
The goal of this article is to elucidate the representation constructions for case frames and referential semantics of verbs of motion used in unmarked extended responses.

\section{A SHORT OVERVIEW OF HAM-ANS}

HAM-ANS is a large German natural language dialog system of both considerable depth and breadth which presently provides access to three different application classes. namely an expert system (hotel reservation situation), a database system (fishery data) and a scene analysis system (traffic scene).

The communicative situations the system handles are characterized as follows:

In the hotel reservation situation the system takes the role of a hotel manager, who tries to persuade the user to book a room. The caller is assumed to have the overali goal of determining as uned to have the overali goal of determining whether the room offered The system must attempt to recognize the users recific desires concerning the room as they are revealed - usually indirectiy - in his utterances and to make use of the various devices available in natural language that permit the roum in ques. tion to be presented in a particularly favorable light le.g. the generation of tendentious descriptions using hedged relative adjectivesl.

In the database application [8] HAM-ANS provides marine scientists with NL access to a fishery database, which contains data collected during international expeditions. in the South Atlantic and the Indian ocean. The complex structured data sets, which consist of cruise data concerning the vessels " voyages, $k r i l l$ biology data, relevant net hauls and oceanographic data are stored in a ret hauls and oceanographic data, are stored in a r PASCALIR data base management system. In the traffic domain we presuppose the following
situational context: The system has observed a 
street intersection and supplies on the phone the user, who is familiar with the scene but cannot about them his remote location, with informat fig. 1). A geometrical description of the observed scene is provided by the image sequence analysis scene is provided by the image sequence analysis system NAOS/MORI0 [9]. In this application our research efforts are concerned with the handiing
of time (tense and temporal adverbs) and the semantics of locomotion verbs (cf. fig. 2 ).

? How many trucks have you seen?

TWO.

? Which trucks have gone by?

A LIGHT-COLORED ONE ON HARTUNGSTREET

? Did a yellow car and a green station wagon turn off first?

NO, A YELLOW CAR TURNED OFF HARTUNGSTREET

ONTO SCHLUETERSTAEET, BUT THE GREEN STATION

DID NOT TURN OFF FIRST.

? Were a young man and an old woman walking

down Schloeterstreet during this time?

YES, AN OLD WOMAN AND A YOUNG MAN.

? Did the green station wagon stop on

Schlueterstreet?

YES

? How many station wagons altogether have stopped?

ONE OF THE THREE ON SCHLUETERSTREET

? What did I just ask you?

YOU ASKED: HOW MANY STATION WAGONS STOPPED

? Did a station wagon drive off?

YES, ONE ON SCHLUETERSTREET

? By the way, the light-colored truck: Did it drive down Schlueterstreet too?

YES

? How many cars are there ill all?

SIXTEEN.

? Is one of these cars blue?

YES. TWD OF THEM ARE KIND OF BLUE

? Did a blue car drive down bath streets?

YES, ONE BLUE CAR

$?$ So long!

F19. 2: A transcript of an actual dialug (translated from German)

\section{REPRESENTATION FORMALISMS FOR THE SEMANTICS OF LOCOMOTION VERBS}

\subsection{THE GEOMETRICAL SCENE DESCRIPTION}

A basic requirement for answering questions about movements that have occurred in real sequences of scenes is an adequate representation of these sequences Not only the shape the centers of sequences. Nut only the shape the centers of gravity col'r, etc. of objects must be represente
otjects

Thls geometrical scene description consists of a combination of automaticaliy generated outputs of the scene analysis processes (insorar as this is presently possiblel and a number of manual augmenpresentiy

The length in time of the scene under consideration is ca. 14 sec. . which corresponds to ca. 360 single TV images. From these 360 images 72 snapstiots are coded in a relational formal.tsm, denoting which objects were observed, the shape of these objects. their current center of gravity and soine other prophot contains information about all objects that are visible at that time For the successive snapshots only changes with respect to the predecessors are recorded, i.e. objects and their descriptions are only entered if they have changed location or appeared in the scene. A trajectory of an object is determined by its different centers of gravity relative to an its different centers of gravity relative to an real TV image sequence this representation is only 2 dimensional and thus provides a birds-eye view 2 dimensiona

\subsection{THE REPRESENTATION LANGUAGES SURF AND DEEP}

The logic-oriented semantic representation languages SURF and DEEP are the central representation formalisms used in HAM-ANS. These languages are designed to be declarative and easily extendable. SURF is the target language of the analysis components and source language for the generation components and thus as close as possible to $\mathrm{NL}$ utterances, whereas DEEP is better suited for the evaluation of utterances on the basis of the system's domain-specific knowledge sources.

Originally SURF and DEEP were designed to represent term and predicate structures which serve as a representation formalism for state descriptions occurring typically in the notel reservation situation. For an adequate representation of the semantics of questions containing verbs the definition of SURF and DEEP was augmented by meta-predicates for marking deep cases. tense and volce adapted from Fillmore $s$ deep case theory [3]. Since events can be existentially quantificin

(6) Did John fly to Hamburg?

(7) Oid John fly to Hamburg three times last week?

SURF and DEEP provide a means of representing quantification of events. A special quantifier $E-A C T$ denotes an existential quantification of events. other quantifiers like those in (7) are currently not available but can easily be included. Examples of SURF and DEEP expressions are shown in the annotated example (cf. fig. 8)

In this paper only some of the features of SURF and DEEP are discussed, see [6] for a more detailed description.

\subsection{THE CASE-FRAME LEXICON}

The case frames for verbs used in the system are stored in the case-frame lexicon [5]. Each entry in the word lexicon for a verb contains a pointer to its applicable case frame which describes the semantics of that verb in terms of case relations.

A case frame is represented as a combination of deep case descriptions specifying for each deep case its name, a marker, whether the deep case is obligatory (0) or optional (F), and the semantic restrictions which are required from a syntactic substructure to fill the deep case (cf. fig. 3 ).

This pointer technique permits the use of a specific case frame for several verbs during the analysis phase witliout predetermining a single process for these verbs during the evaluation of whole utterances. For verbs, with different referential semantics, e.g. 'to accelerate' and to stop", a single case frame, namely that speci. fying an obligatory AGENT of type 'vehicle', and a applied during the analysis phase.

Case frames are formulated in SURF so that the checking of the semantic restrictions can be accomplished by the inference rules usually applied during the evaluation of a complete utter ance; The selectional restriction that e.g.j the NP ; car describe an object of the ciass of fill the agent role of the verb to stop'. can be 
verified because of the transitivity of the superset relation in the conceptual semantic net.

In the case-frame lexicon the case frames are not recorded in the form shown in fig. 3, but rather are represented as constructor calls for building

$[r]-s:$ agent:

ld-1: role-marker: 0

restrictions:

(1 ambda: $x 1$ (af-a: ISA $\times 1$ VEHICLE])]

objective:

source:

locative

(d-l: role-marker: F

restrictions

[lambda: $x 1$ (af-a: ISA $\times 1$ THOROUGHFARE])] goal:

time

path:

instrument: :

Fig. 3: Case frames for verbs of type 'to stop'

a case frame according to the actual syntax definition of SURF. This guarantees that all possible modifications of SURF are immediately present in the case frames.

\subsection{OBJECT-ORIENTED REPRESENTATION OF MOTION CONCEPTS}

In object-oriented programming languages programming is more or less the activity of creating a world of entities called objects and of specifying a set of generic operations that can be performed on them. objects can communicate with each other by sending and receiving messages Essentially. running program means that the object sends message to an object (possibly to itself) which in turn sends a message etc until the required task is oriented style is that it lends itself to a particularly simple and lucid kind of modularity.

\subsubsection{THE FLAVOR SYSTEM}

The Flavor system [2] [13] is an implementation of the language reatures that support object-oriented programming. Two kinds of objects exist in a Flavor system, ramely one called flavor and the other instance of a flavor. A flavor represents a generic object and an instance an individual realiza tion of a gueric object. It is possible to send tiones of called. Flavors are organized in a drected graph called the flavor graph There is one designated flavor. the vanilia flavur. which corresponds to the thing frame in FRL [10]. Since the heritage of information for each flavor is provided by the flavor graph, it is necessary to specify for each newly defined flavor its location in the graph by naming its direct predecessors (its superflavors). The information contained in a flavor is a combination of all the information inherited from its superflavors and the added information given by its own definition. The added information $c$ an also over ride, augment or modify the inherited information.

This is one dimension of the information contained in a flavor: owned or inherited. Another is the declarative/procedural distinction. The declarative knowledge of a flavor is stared in variables of different kinds whereas procedural. knowledge is encoded in so.called methods.

One kind of variable . the instance variable - is used to give instances of the same generic object their individual information. The ather kind the class variable . is owned by a flavor. can be 'bequeathed to other flavors, and accessed by any object in the flavor system. However a flavor is only allowed to change a value of a class variable, if it owns this variable.

Methods are function definitions that implement the operations defined for each flavor. The combination of methods from different flavors is called mixing flavors.

In comparison with FRL the Flavor system has mainly three distinguishing features:

- The 'A kind of' slot in FRL serves both for establishing an inheritance hierarchy and for connecting instances to superclasses, i.e. no clear distinction is made between generic frames and instances. On the other hand the flavor graph is built by specifying the flavor graph is built by specifying the superflavors for each flavor, insta
created by the make-instance-method.

- Because the distinction between generic frames and instances is not made in FRL there is also no distinction between instance variables and class variables. In the flavor sys. tem the semantics of variables is more cem the semantics of variables is more clearly defined in that instance variables variables can only be modified in flavors.

- Frames in FRL are passive data structures whereas flavors passive data structures. whereas flavors can be lre-lactivated: they are declarative and procedural at the same time and hence are entities which are better suited for as formalisms for representing common knowledge (cf. [2]).

Although the flavor system is a tool for the development of large software systems and not a knowledge representation language. it includes the basic concepts for the rapid design of specific knowledge representation formalisms. In contrast to a full-fledged knowledge representation language this approach requires some additional programming in the beginning. but it avoids any permanent overhead for features which are superfluous for the task at hand.

\subsubsection{THE MOTION CONCEPT HIERARCHY}

The Flavor system is used in HAM-ANS for representing a specialization hierarchy of motion hierarchy is the motion concept MOVE Descendants in the tree e motion concept TURN inherit the declarative and procedural'information contained

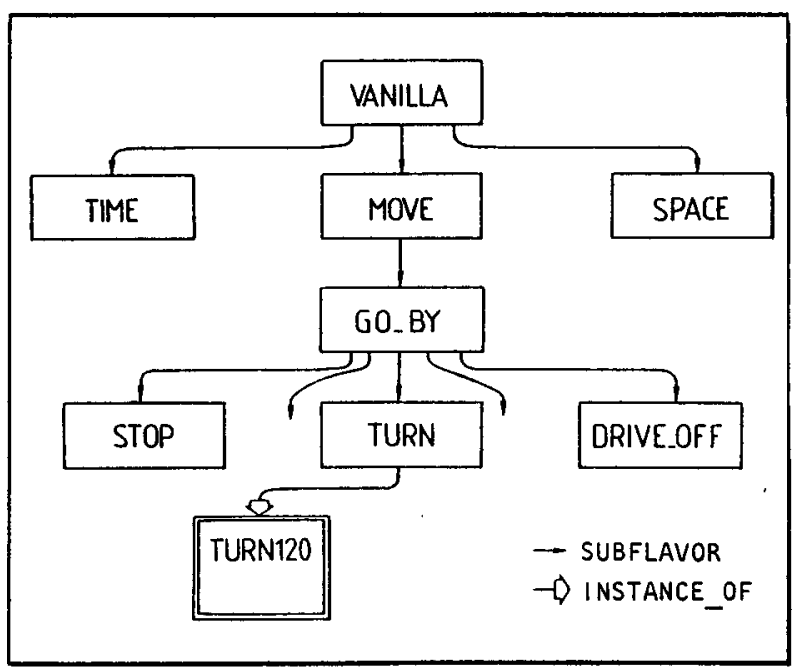

Fig. 4: The notion concept hierarchy 


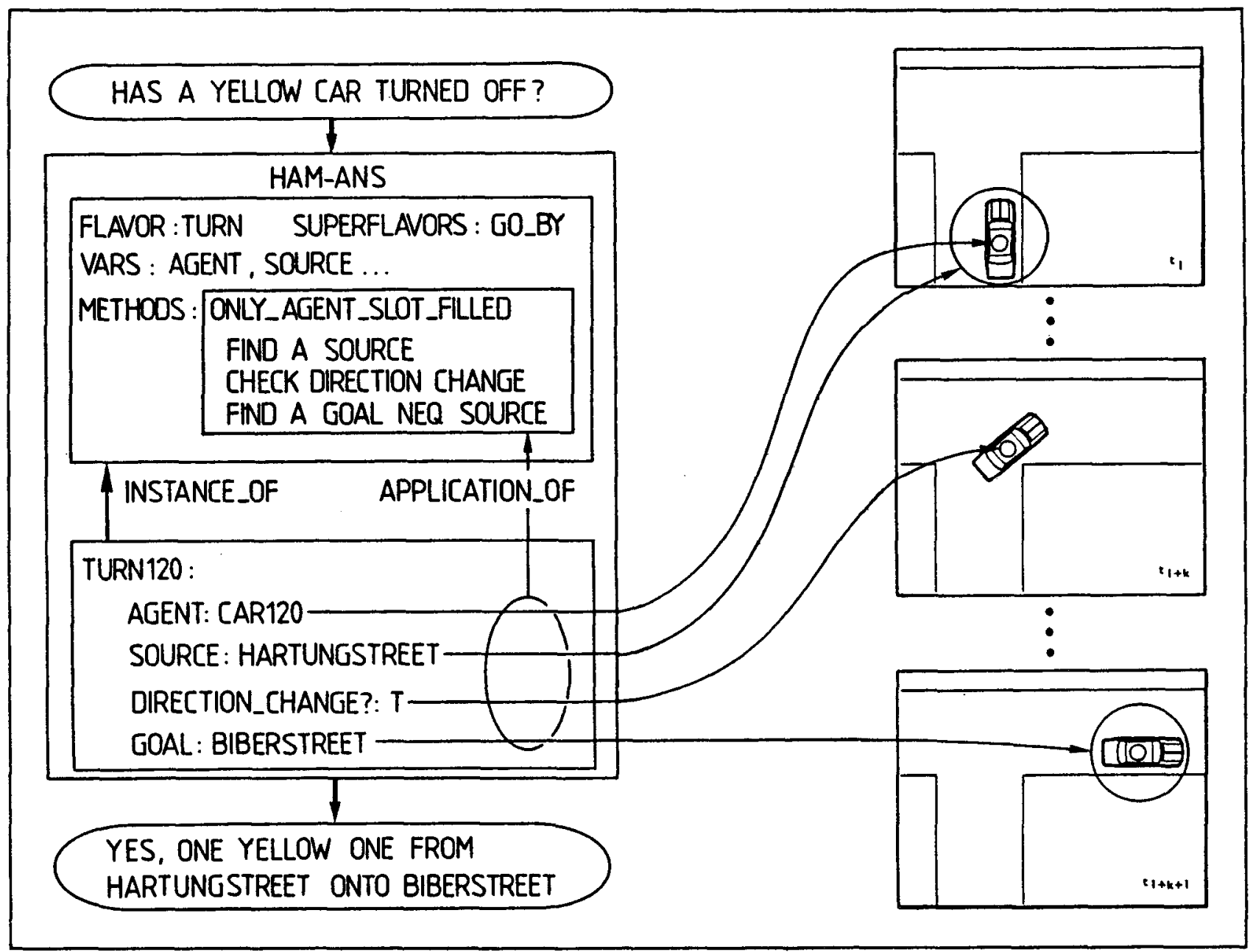

Fig. 5: Case slot filling as side effect of visual seareh

in their parents. Instance variables comprise information about the deep cases associated with the motion concept as well as information needed and extracted by methods. The methods are responsible for checking the referential semantics of the motion concepts. Instances of a flavor denote specific events in the domain of discourse that could be verified by the application of the methods.

The methods of the additionally defined flavors TIME and SPACE are responsible for temporal and spatial computations. Instances of these flavors determine tho temporal and spatial description of the actual scene. the length of the scene in time. the rumber of snapshots, the spatial extent, etc.

The task of cliecking the truth value of the proposition in i user's question is accomplished through messaile passing. These messages include: creating in.tarices of motion concepts, e.g. CURNi20, instintiating deep case slots specified ill the quistion. and activating appropriate methods.

Let's now concider the example given in fig. 5 in more detail. ince only the AGENT was speciried in the question, the selected method is ONLY AGENT SLiT_ILLED. After determining an interval of consideration this method calis further mithods, namely FIND A SOURCE, DIRECTION CHANGE and FIND_A_GOAL NEO_SOURCE. DIRECTION CHALGE is a special method of the flavor TURN. The first and last methods are inherited lef. fig. G) from flavor GO_BY because they are also needed in that flavor for answering questions like: Has the yellow car driven from Biberstreet to Hartungstreet?

FIND_A_SOURCE identifies the first entry of the agen $\bar{t} \cdot \bar{s}$ trajectory in the interval of consideration and checks which of the objects of the static background these coordinates belong to. For this test only those static objects are selected that satisfy the selectional restrictions for the source slot specified in the case-frame lexicon. If the test succeeds for an object, the name of this object is stored in the source slot. DIRECTION CHANGE now follows the agent's trajec: tory looking for a significant change of direc. tion looking for a significant change of dilive FIND A GOAL NEO SOURCE is tried. This method searches for a point on the trajectory which is not inside the object identified in the source slot. If there is such a point, the same selectional check as for the source slot is executed for the possible goal object. The successful. application of these methods yields a fully instantiated flavor instance e.g. TURN 120 lcf fig. 7 ).

\section{AN EXAMPLE OF THE PROCESSING OF AN UTTERANCE}

The processing of a user's utterance may be illus trated by an example taken from the dialog in fig.

USER: Which trucks have gone by? HAM-ANS: A YELLOW ONE DN HARTUNGSTREET. 

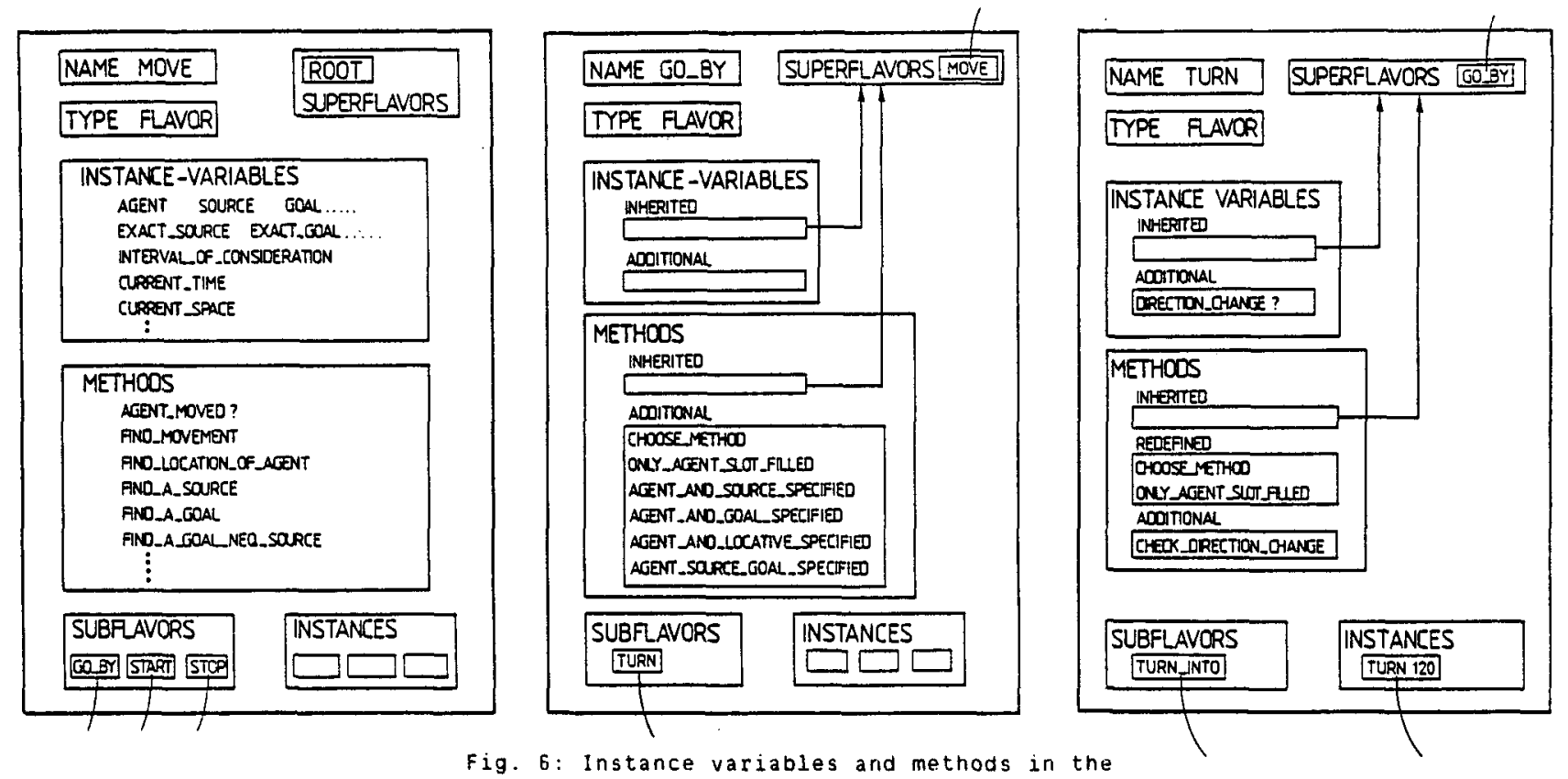

Fig. 6: Instance variables and methods in the motion concept hierarchy

The following discussion of some of the processing phases can best be understood if continual reference is made to fig. 8 , which shows a traced version of the example.

The processing of a user's NL input starts with a rather elaborate lexical and morphological analysis - a process which on the one hand reduces single words to their canonical forms with their morphological and syntactic features (e.g. gender, person, number) and on the other hand recognizes syntagmatic groups of woras and discontinuous verb constituents. transforming them according to predefined rules.

The generated structure the preterminal string (not shown in fig. 8) forms the input to the parser. The syntactic analysis consists of two ATN-definitions of syntactic categories the same noun ohrases and prepositional phrases. One of

\begin{tabular}{|c|c|c|}
\hline NAME TURN 120 & \multicolumn{2}{|l|}{ INSTANCE_OF } \\
\hline \multicolumn{3}{|l|}{ TYPE INSTANCE } \\
\hline \multicolumn{3}{|c|}{ INSTANCE VARIABLES } \\
\hline NAME & VALUE & FILLED_BY_METHOO \\
\hline AGENT & CAR 20 & \multirow{3}{*}{ MAKE INSTANCE } \\
\hline EURRENT_TIME & TSO 128 & \\
\hline CURRENT_SPACE & SSD 128 & \\
\hline \multicolumn{3}{|c|}{ INTERVAL_OF_CONSOERATION（21.64) } \\
\hline SOURCE & BIBERSTREET & \multirow{2}{*}{ FIND_A_SOURCE } \\
\hline EXACT_SOURCE & $(50.70)$ & \\
\hline OIREETION_CHANGE? & CHECKL & CHECK_DIRECTION_CHANGE \\
\hline $\begin{array}{l}\text { GOAL } \\
\text { EXACT_LOAL }\end{array}$ & $\left.\begin{array}{c}\text { HARTUNGSTREET } \\
(300.100)\end{array}\right\} \begin{array}{c}\text { FII } \\
\mathrm{SO}\end{array}$ & $\begin{array}{l}\text { FIND_A_GOAL_NEQ } \\
\text { SOURCE }\end{array}$ \\
\hline
\end{tabular}

Fig. 7: An instance of TURN these strategies - always applied for sentences with copula verbs - uses a surface grammar to cope with word order variations. The other is a casedriven analysis strategy which is used for sentences containing verbs with an associated case tences
frame.

Since in the example the verb 'to go by' has a case frame the second strategy is applied. After an access to the case-frame lexicon the case frame is constructed. This case frame is used to guide the parsing in the following manner: The algorithm stituents that are possible candidates for a deep stituents that are possible candidates for a deep those constituents that are possible candidates for optional deep cases. When the input is comfor optional deep cases. When the input is compliled the process ends.

The test for determining if a syntactic constituent is a possible candidate to fill a specific deep case is divided into a syntactic and a seman$t i c$ check. The syntactic check requires e.g. that in order to fill the agent role a constituent must contain the attribute nominative isentence in active voicel and that its number must correspond to that of the verb. The semantic check requires that the noun of the constituent fulfill the semantic restrictions specified for the specific deep case. This is accomplished through the building of a SURF expression for the constituent the transformation of this expression into expression on the basis of the conceptual net.

In our example only the agent case is marked as obligatory and the noun phrase 'which trucks fulfills both the syntactic and semantic requirements to fill this slot. Since no other syntactic con-
stituents are encountered. the complete suRf representation is constructed.

The structure is rormalized into a DEEP structure. One of the maln tasks of this process is the determination of the scope of quantifiers. The algorithm used for this purpose is modelled after the one described by Hendrix [4]; it takes into account the relative strength of natural language quantifiers (e.g. 'a' 'both') and question opera.. tors (e.g. which' how many') The strength is determined by a numeric value which in some cases determined by a numeric value, which in some cases noun weaker than the more specific quantifier both. Is 


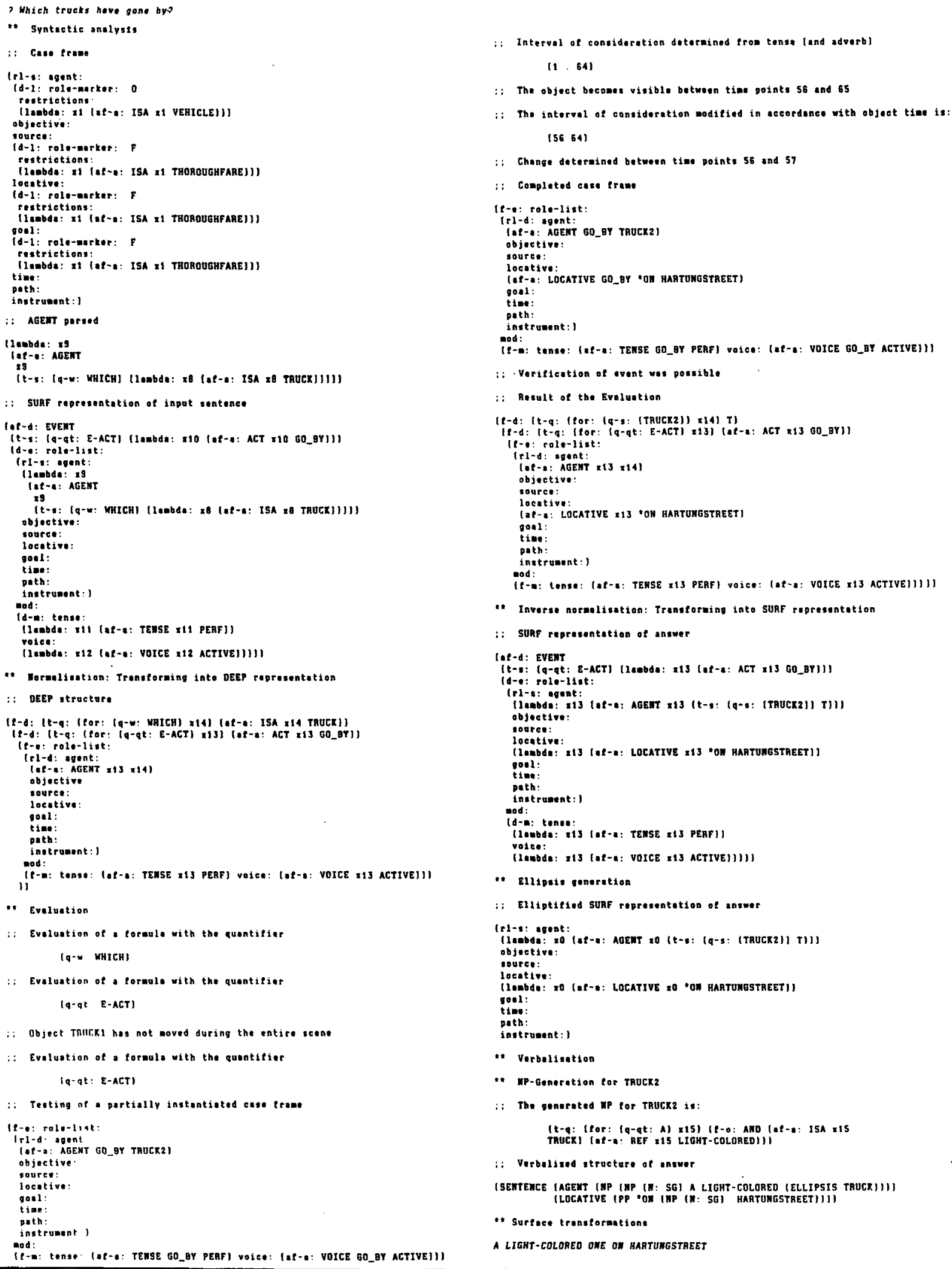

Fig. 8: Annotated example interaction 
Since, in the example discussed, the question operator 'which' is stronger than the existential rearranged.

The task of evaluating a DEEP formula is governed by a generate and test strategy. Generate and test procedures can be viewed as being activated by pattern-directed invocation and differ from oach other in that the generate procedures assign internal object identifiers to variables in DEEP internal object identifiers to variables in DEE formulas, while the test procedures yield two values, the first of which is either a fully mula or a modified formula. and the second of mula or a modified formula, and the second of which indicates the truth value of the input for-
mula in the range $[0,1]$. In the interpretation phase these two processes interact in such a way that a test attempt activates generate procedures which in turn call test procedures and so on.

A closer look at our example shows that after the first test attempt has discovered a structure containing a variable - in this case the term package of generate procedures is activated to produce the set of object identifiers denoting the referential set of objects that are trucks - here TRUCKI and TRUCK2. The rest of the formula is then recursively sent to a test process with the then recursively sent to a test process with the ence set for trucks one after the other.

The next formula to be tested requires the generation of a set of instances of the type GO BY. since events are not represented in fülyy from the geometrical scene description, a special set of procedures - the methods specified in the verb flavor hierarchy - is activated. (see section 3.4 .2 for how this process functions.)

A verification of an event GO_oy is possible only for TRUCK2. The additional information extracted during the process of visual search - the specific location of the event - is recorded in the locative slot.

During the formation of the result of the evaluation, the system, guided by general heuristics. decides whether the additional detail will cause too great a complexity in the answer or not [11]. In this case the complexity is suitable and the location will be mentioned in the answer and the is defined as quantifier that causes a description of a set of objects to be returned (instead of a truth valuel. Thus the set ceference objects for which the proposition in stituted for the noun phrase which trucks".

The resulting DEEP expression is transformed by the inverse normalization process into a SURF expression. In order to verbalize extended as possible the ellipsis generation process elides those parts of the semantic representation of complete responses that are identical to the stored representation of the question [ 7 ]

The verbalization component produces a string of canonical words and their grammatical features using translation rules attached to the various categories of SURF expressions. A special subcomponent provides for the generation of noun phrases as descriptions of domain individuals, in our example TRUCK2. In this case the NP-generator decides not to generate a definite description since neither the system nor the user has already referred to TRUCK 2 in the previous dialog and the oxistence of TRUCK2 as a moving object is not implied by the existential assumptions supplied by the a priori user model (cf. [7]). Ingtead, the indefinite NP a light-colored truck is generated using the property lignt-colored as an initial characterization.

Finally the 'surface trangformation' component [1] pronominalizes the noun truck and yields standard word order of the utterance and the
correctiy inflected forms of the canonical words.

\section{CONCLUSION}

We have attempted to show that case role filling for the construction of an unmarked extended response can be regarded as a side effect of the visual search necessary to answer questions referring to a visualiy present domain of discourse. A new method for the representation of the referen tial semantics associated with locomotion verbs has been presented in the framework of object oriented programming based on the flavor system. he appraach presented has been useful in extendsystem HAM-ANS as an interface to a vision system.

\section{REFERENCES}

[1] BUSEMANN, S.: Problems involving the automatic generation of utterances in German. Memo ANS-8, Research Unit for Information

[2] DI PRIMIO, F. CHRISTALLER, T.: A poor man's flavor system. Working paper No. 47, ISSCO. Univ. de Geneve. 1963 .

[3] FILlMORE, C. J.: The case for case. In: Bach, E. Harms $\dot{R} T$ (eds ): Universals in linguistic theory. Holt. Rinenart \& winston. 1968 , pp. 1-88

[4] HENDRIX, G. G.: Semantic aspects of translation. In: Waiker, D. E. (ed.): Understanding spoken language.
i978. pp. 193-228

[5] HOEPPNER, W.: ATN-Steuerung durch Kasusrahmen. In: Wahlster, W. (ed.): GWAI-82. Proc. 6th German Workshop on AI. Berlin: Springer 1982. pp. 215-226.

[5] HOEPPNER, W. CHRISTALLER, TH MARQURGER, HTER, W.: Beyond doma in independence: Experience with the development of a German ence with the development of a German language access system to highly diverse Karisruhe 1983 . pp. $588-594$.

[7] JAMESON. A., WAHLSTER, W.: User modelling in anaphora generation: Eiiipsis and definite description. In: Proc. ECAI-82, Orsay 1982, pp. $222-227$

[8] MARBURGER, H. NEBEL, B.:

Natuerlichsprachlicheir Datenbankzugang mit HAM-ANS: chsprachlicher oatenbankzugang mit HAM-ANS: Syntaktische Korrespondenz, natuerlichspraModell des Diskursbereichs. In: Kupka I. (ed.): GI-13. Jahrestagung. (To appear)

[9] NEUMANN B.: Towards natural language description of real-world image sequences. Berlin: Springer, ig82, pp. 349-358.

[10] ROBERTS, R.B., GOLDSTEIN, I.P.: The FRL manual. AI Memo 609, AI Lab.. MIT, Cambridge. 1977.

[1 1 ] WAHLSTER, W. MARBURGER, H.: JAMESON, A.: BUSEMANN, S.: Over-answering yes-no questions: Extended responses in a NL oth IJCAI. Karlsruhe 1983, pp. 643-666.

[12] WEBBER, B., JOSHI, A., MAYS, E.. MCKEOWN, K.: Extended natural language database interaction. In: Int. J. Computers and Mathematics. Spring 1983

[13] WEINREB, D., MOON, D.: Lisp Machine Manual (hth ed.). MIT, 1981 . 